\title{
Flow is not perfusion, and perfusion is not function: ancillary testing for the diagnosis of brain death
}

\author{
Guillaume Plourde, MD, PhD, FRCPC • Joel Neves Briard, MD • \\ Sam D. Shemie, MD • Jai Jai Shiva Shankar, MD, DM, MSc, FRCPC • \\ Michaël Chassé, MD, PhD, FRCPC
}

Received: 7 January 2021 / Revised: 6 February 2021 / Accepted: 14 February 2021/Published online: 4 May 2021

(C) The Author(s) 2021

Death by neurologic criteria (DNC), commonly known as "brain death" (BD), is defined as the irreversible cessation of consciousness and brainstem responses in a plausible clinical context and in the absence of significant confounders. $^{1-5}$ Stated differently, it represents the definitive arrest of brainstem function, arising most commonly from a primary devastating brain injury. Hence, the cornerstone of $\mathrm{BD} / \mathrm{DNC}$ determination is showing dysfunction of the ascending reticular activating

G. Plourde, MD, PhD, FRCPC

Division of Critical Care, Department of Medicine, Centre hospitalier de l'Université de Montréal, Montréal, QC, Canada

J. N. Briard, MD

Department of Neuroscience, Université de Montréal, Montréal, QC, Canada

\section{S. D. Shemie, MD}

Division of Critical Care, Montréal Children's Hospital, Research Institute of the McGill University Health Centre, Montréal, QC, Canada

Deceased Organ Donation, Canadian Blood Services, Ottawa, ON, Canada

\section{J. J. S. Shankar, MD, DM, MSc, FRCPC}

Department of Radiology, University of Manitoba, Winnipeg, MB, Canada

\section{Chassé, MD, PhD, FRCPC ( $\varangle)$}

Division of Critical Care, Department of Medicine, Centre hospitalier de l'Université de Montréal, Montréal, QC, Canada

e-mail: michael.chasse@umontreal.ca

Centre hospitalier de l'Université de Montréal Research Center (CRCHUM), 900 rue Saint-Denis, Montréal, QC H2X 3H8, Canada system (coma) and brainstem nuclei (absence of cranial nerve reflexes, including irreversible central apnea) through physical examination.

Since its first description in $1968,{ }^{6}$ our comprehension of neurologic death has evolved in parallel with the development of novel neuroimaging modalities. Despite initial design as adjuncts to physical examination, these tests are now often employed to evaluate brain blood flow, perfusion, and function in the event of an inconclusive clinical exam. We define flow as the movement of blood detected by opacification of major brain vessels (arteries and veins) in blood flow studies, whereas perfusion implies capillary-level flow in the target tissue (in this case the brain). Both flow and perfusion enable an organized clinical brainstem neuronal function, as manifested by consciousness, organized motor responses, and brainstem reflexes. While these parameters are distinct physiologically, there has been a certain degree of misnaming or misinterpretation in the literature, with the terms "flow", "perfusion", and "function" being used interchangeably. ${ }^{3,4,7-9}$ For instance, perfusion is frequently used to designate flow and may erroneously conclude that the presence of flow or perfusion are definitive signs of function. Any brain function requires perfusion, and perfusion requires flow. Nevertheless, the detection of flow does not infer perfusion, and the detection of perfusion does not necessarily infer function. Clear definitions of flow, perfusion, and function might improve understanding of ancillary tests, promote further research to identify an ideal test, and standardize test use in patient care.

To illustrate the fundamental differences between these three parameters, we propose a graphic conceptual model involving a flower being watered by a hose (Figure). In this model, the flower represents the cerebrum and brainstem. To reach the flower, water must first move through the hose 
FIGURE Conceptual model of brain blood flow, perfusion, and function. (C2020 Devon Medical Art LLC.Modèle conceptuel de la circulation sanguine, la perfusion, et la fonction cérébrales. (C2020 Devon Medical Art LLC.

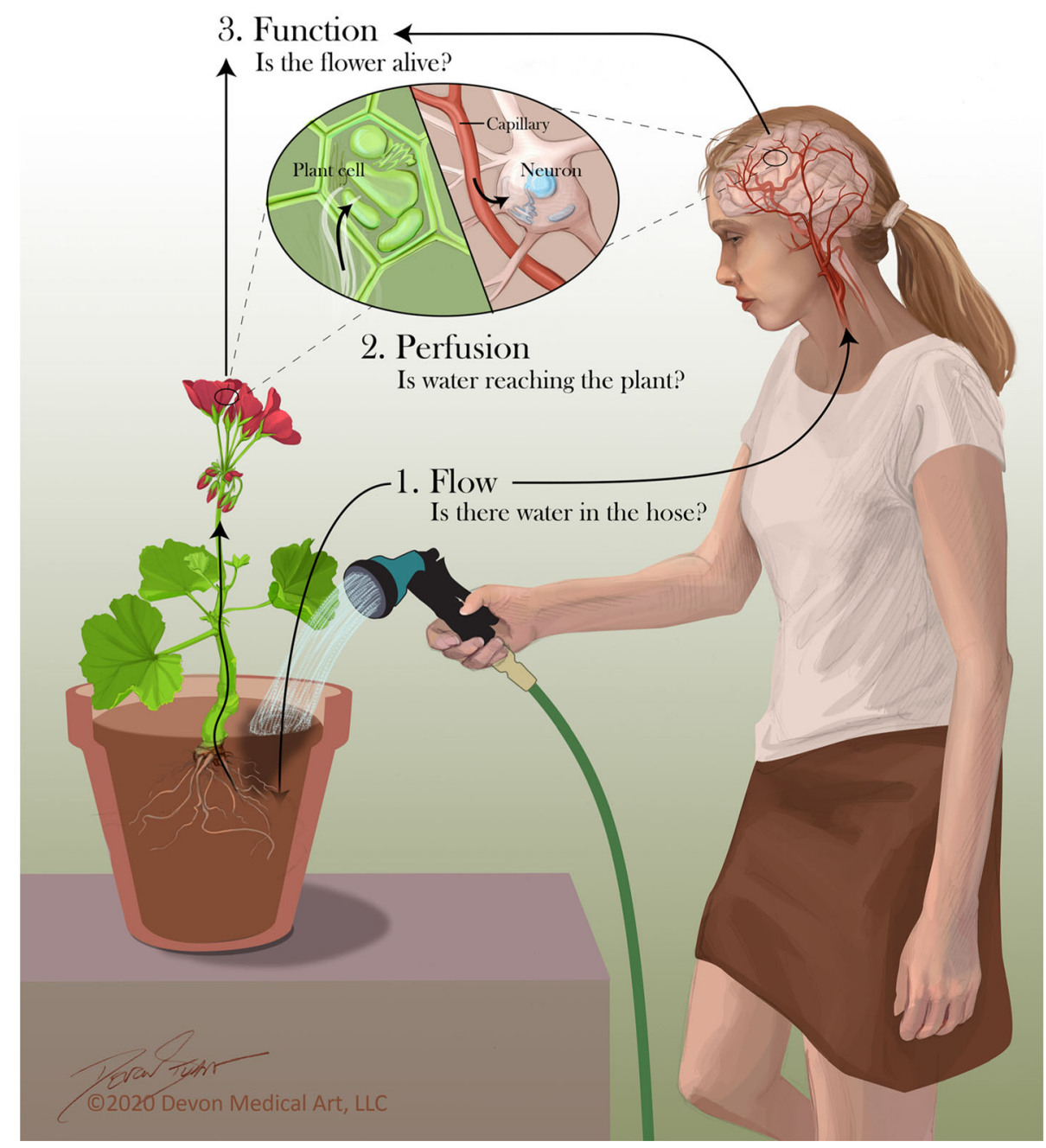

(flow) and then be projected into the flower (perfusion). In the case of $\mathrm{BD} / \mathrm{DNC}$, our interest is to determine if the cerebrum and brainstem have a cessation of function, which is equivalent to showing that the flower is dead, regardless of whether water flows in the hose or is perfused into the flower.

Recent articles have extensively reviewed ancillary testing for neurologic death. ${ }^{1,7,10}$ Even after the recent World Brain Death Project criteria for BD/DNC publication by Greer and colleagues, we feel ancillary testing use and interpretation remain misunderstood and controversial. ${ }^{11}$ We hereby propose a short critical appraisal of some of these tests while providing a deeper conceptual and physiologic understanding of the distinctions between brain flow, perfusion, and function (Table 1). We also propose actualized definitions of flow, perfusion, and function based on our conceptual model (Table 2).

\section{Is there cerebral blood flow? (Is there water in the hose?)}

Flow is defined as the steady movement of a fluid through the tissue blood vessels. Cerebral blood flow is necessary for perfusion, and thereby, for function. In the context of $\mathrm{BD} / \mathrm{DNC}$, unequivocal demonstration of the absence of flow is consistent with neurologic death, ${ }^{3}$ but the presence of flow itself is insufficient to prove cerebral perfusion and function. Traditionally, four-vessel conventional angiography, transcranial Doppler ultrasound, and CT/ MR-angiography have been employed as tools to detect brain flow. Despite having good sensitivity for flow, these tests are subject to various caveats. For instance, false negative cases have been described where brain flow was documented on CT-angiography (CTA) despite conventional angiography showing an absence of opacification in brain vasculature. ${ }^{12}$ The presence of blood flow (water in the hose) is relatively easy to show, 
TABLE 1 Empiric qualitative comparison of ancillary tests for brain death/death by neurologic criteria

\begin{tabular}{|c|c|c|c|c|c|}
\hline & \multicolumn{2}{|c|}{ Flow } & \multicolumn{2}{|c|}{ Perfusion } & \multirow{2}{*}{ Function } \\
\hline & Cerebrum & Brainstem & Cerebrum & Brainstem & \\
\hline 4-vessel angiography & $\oplus$ & $\oplus$ & $\ominus$ & $\ominus$ & $\otimes$ \\
\hline Transcranial Doppler & $\oplus$ & $0^{*}$ & $\otimes$ & $\otimes$ & $\otimes$ \\
\hline CT-angiography & $\bigoplus \oplus$ & $\bigoplus$ & $\ominus$ & $\otimes$ & $\otimes$ \\
\hline CT-perfusion & $(\oplus \infty)$ & $(\oplus \infty \oplus)$ & $(\oplus \infty \infty)$ & $(\oplus \infty \infty)$ & $\otimes$ \\
\hline Radionuclide imaging & $\oplus \oplus$ & $\ominus$ & $\infty$ & $\ominus$ & $\ominus$ \\
\hline Electroencephalography & $\otimes$ & $\otimes$ & $\otimes$ & $\otimes$ & $\ominus$ \\
\hline Evoked Potentials & $\otimes$ & $\otimes$ & $\otimes$ & $\otimes$ & $\ominus$ \\
\hline Clinical examination & $\otimes$ & $\otimes$ & $\otimes$ & $\otimes$ & $\oplus \infty \oplus$ \\
\hline
\end{tabular}

$\bigotimes=$ not useful

$\ominus=$ may be useful (conditions generally apply)

$\bigoplus=$ useful

( ) = under study

* Transcranial Doppler can evaluate flow in the vertebral arteries and the basilar artery (acoustic window permitting).

CT: computed tomography.

Empiric qualitative comparison of ancillary tests and clinical examination for brain death/death by neurological criteria determination.

CT-perfusion imaging is actually under study in the prospective INDex-CTP trial (ClinicalTrials.gov: NCT03098511).

but remains sometimes inconclusive, with further imaging necessary to evaluate perfusion or function.

\section{In the presence of flow, is there brain perfusion? (Is water reaching the flower?)}

Whereas brain flow is normally investigated in the major brain arteries and veins, evaluation of brain perfusion implies inspection of the cerebral capillary bed. There has been a growing interest in previous years for perfusion imaging in context of $\mathrm{BD} / \mathrm{DNC}$, but these methods remain to be validated. ${ }^{9,13}$ Perfusion imaging, such as the CTperfusion scan (CTP), has many potential advantages over other neuroimaging modalities: it is widely available, less operator-dependent and may even provide a more timely diagnosis than CTA. ${ }^{13}$ Indeed, some authors reported that $\mathrm{BD} / \mathrm{DNC}$ declaration has been delayed by more than $24 \mathrm{hr}$ after an inconclusive CTA, while there was concurrent evidence of brainstem "death" on the CTP. ${ }^{13}$ This is particularly true in the presence of residual flow of unclear certainty. Whereas flow imaging such as CTA is useful to evaluate the presence or absence of contrast (flow) in large brain vessels, perfusion imaging such as CTP has the potential to provide a quantitative and qualitative assessment of cerebral blood volume and flow in various regions of the brain. ${ }^{14}$ Moreover, CTP may help diagnose a primary infratentorial brainstem catastrophe (such as brainstem infarction), a relatively rare scenario in which an isolated perfusion deficit in the brainstem causes clinical cessation of brainstem function despite the preserved flow and perfusion in other brain areas. ${ }^{13}$ Its main caveat remains lack of validation, but pilot studies show an excellent profile of sensitivity, specificity and predictive values compared with CTA and clinical examination. $^{13,15,16}$ Large-scale validation of CTP's diagnostic accuracy in $\mathrm{BD} / \mathrm{DNC}$ is ongoing through the prospective INDex-CTP study (clinicaltrials.gov: NCT03098511). 
TABLE 2 Proposed definitions related to death by neurologic criteria

\begin{tabular}{llr}
\hline & Proposed actualized definition & Conceptual model correlate \\
\hline Flow & $\begin{array}{l}\text { Movement of blood detected by opacification of major brain* vessels (arteries and } \\
\text { veins) in blood flow studies }\end{array}$ & Is there water in the hose? \\
Perfusion & $\begin{array}{l}\text { Capillary-level flow in the target tissue (in this case brain, specifically brainstem) } \\
\text { Function }\end{array}$ & $\begin{array}{l}\text { Organized clinical brainstem neuronal function as manifest by consciousness and } \\
\text { brainstem reflexes }\end{array}$ \\
\hline
\end{tabular}

*Brain is defined as the combination of the cerebrum and the brainstem.

Proposed actualized definitions of the concepts of brain blood flow, perfusion, and function in the light of our new conceptual model. These actualized definitions put the emphasis on the physiologic differences between the three concepts to avoid potential misinterpretation or misnaming.

\section{If there is presence of perfusion, is there brain function? (Is the flower alive?)}

As clinicians evaluating $\mathrm{BD} / \mathrm{DNC}$, our main focus is the assessment of function, which may be defined as the integration of a continuous neuronal input and output in the cerebrum and brainstem in response to physiologic stimuli. This has been widely accepted internationally based on a clinical evaluation documenting the absence of capacity for consciousness and brainstem areflexia. ${ }^{17}$ The recently released 2020 World Brain Death Project criteria for BD/ DNC further specified that neurologic death diagnosis only requires the demonstration of the absence of brainstem function essential for consciousness and other vital functions such as autonomous breathing (Table 1). ${ }^{11}$ Hence, physically examining the brainstem remains the gold standard method for determining $\mathrm{BD} / \mathrm{DNC}$. Unfortunately, it is often limited by confounding factors, such as intoxication and major facial trauma, and is subject to inter-rater variability. ${ }^{10,18,19}$ Throughout the years, many ancillary tests have focused on functional evaluation, such as electroencephalography, evoked potentials, and radionuclide imaging. They mostly provide an objective and qualitative evaluation of cerebral cortical function. That being said, they are usually limited by the same confounding factors as the clinical exam. In light of these limitations, some authors consider studying cerebral perfusion with $99 \mathrm{mTc}$-HMPAO single photon emission CT (SPECT) the ideal ancillary test. $^{7}$ Nevertheless, HMPAO-SPECT has limited availability and spatial resolution, requires a long acquisition time, and is subject to various artefacts that may also hinder its interpretation, notably at the brainstem level. A clinical example of these caveats would be a patient with facial trauma (clinical confounder mandating ancillary testing) hospitalized in a regional general critical care unit (limited access to advanced neuroimaging) in acute intracranial hypertension with unstable heart-brain axis (limited tolerance to long imaging acquisition time) following an intracranial hemorrhage due to a ruptured basilar aneurysm. Such a patient would still represent a considerable challenge and illustrates the complexity of an accurate and timely BD/DNC determination.

\section{Implications for the future of ancillary testing in BD/ DNC}

Beyond improving nomenclature, the distinctions between flow, perfusion, and function have implications for the future of ancillary testing in $\mathrm{BD} / \mathrm{DNC}$ determination. Once thoroughly validated in high-quality diagnostic accuracy studies, novel ancillary tests that assess brainstem perfusion (e.g., CTP) or function may expedite BD/DNC determination in certain patients. For instance, these tests could prove particularly useful for patients with isolated brainstem injury who undergo ancillary testing for clinical indications (e.g., confounders to clinical examination) or because of legal regulations (e.g., Germany requires ancillary testing for all patients who are suspected of $\mathrm{BD} /$ DNC due to an infratentorial lesion). ${ }^{20}$ As noted previously, there is evidence that supratentorial blood flow can persist in patients that fulfill all clinical criteria for $\mathrm{BD} / \mathrm{DNC}$ and have absent brainstem perfusion on CTP. ${ }^{13}$ Based on case reports and series, other authors have hypothesized that patients with isolated brainstem death eventually progress to whole-BD because of severe intracranial hypertension from obstructive hydrocephalus, further compounded by the mass effect on the vein of Galen. ${ }^{21,22}$ Although this process may occur in a matter of hours in some patients, progression to whole-BD may be a matter of weeks in patients who have undergone decompressive craniectomy. ${ }^{22}$ Ancillary tests thoroughly validated for brainstem perfusion or function may therefore permit prompt BD/DNC diagnosis in such patients, avoiding delays incurred by the necessity to prove absent 
cerebral blood flow and permitting rapid evaluation for organ donation.

\section{Conclusion}

Death by neurologic criteria is fundamentally a clinical determination based on the permanent absence of capacity for consciousness and brainstem areflexia. In the presence of unresolvable confounders or inability to complete clinical evaluation, reliance on ancillary testing remains a challenging requirement. We propose a conceptual framework to clearly distinguish brain flow, perfusion, and function. Brain function requires perfusion and perfusion requires flow. Nevertheless, the presence of flow does not infer perfusion and the presence of perfusion does not infer function. The ideal ancillary test should be rapid, safe, readily available, non-invasive, inexpensive, and not susceptible to confounding factors. ${ }^{7}$ In addition, the ideal test should be as close as possible to a nonconfounded function assessment and should refrain from using flow as a surrogate. Such an ideal test is not yet validated.

\section{Circulation ne veut pas dire perfusion, et perfusion ne signifie pas fonction : les tests complémentaires pour le diagnostic de la mort cérébrale}

Le décès déterminé par des critères neurologiques (DDN), communément appelé «mort cérébrale » $(\mathrm{MC})$, est défini comme la cessation irréversible de la conscience et des réponses du tronc cérébral dans un contexte clinique plausible et en l'absence de facteurs de confusion significatifs. ${ }^{1}{ }^{5}$ En d'autres mots, il s'agit de l'arrêt définitif de la fonction du tronc cérébral, résultant le plus souvent d'une lésion cérébrale primaire dévastatrice. Par conséquent, la pierre angulaire de la détermination de la MC/DDN consiste à démontrer le dysfonctionnement du système d'activation réticulaire ascendant (coma) et des noyaux du tronc cérébral (absence de réflexes des nerfs crâniens, y compris une apnée centrale irréversible) par un examen physique.

Depuis sa première description en $1968,{ }^{6}$ notre compréhension de la mort neurologique a évolué en parallèle au développement de nouvelles modalités de neuroimagerie. Malgré leur conception initiale comme complément à l'examen physique, ces tests sont aujourd'hui souvent utilisés pour évaluer la circulation sanguine, la perfusion, et la fonction du cerveau en cas d'examen clinique non concluant. Nous définissons la circulation comme le mouvement du sang détecté par l'opacification des vaisseaux majeurs du cerveau (artères et veines) dans les études de flux sanguin, tandis que la perfusion implique une circulation au niveau des capillaires dans le tissu cible (dans ce cas, le cerveau). La circulation et la perfusion sont toutes deux nécessaires à une fonction neuronale clinique organisée du tronc cérébral, telle que démontrée par la conscience, les réponses motrices organisées, et les réflexes du tronc cérébral. Bien que ces paramètres soient distincts d'un point de vue physiologique, la littérature comporte plusieurs cas d'inexactitude dans la nomenclature et son interprétation, les termes « circulation », «perfusion » et «fonction » étant utilisés de façon interchangeable. ${ }^{3,4,7-9}$ Par exemple, on utilise fréquemment le terme de perfusion pour désigner la circulation, ce qui peut mener à la conclusion erronée que la présence de circulation ou de perfusion sont des signes définitifs de fonction. Toute fonction cérébrale nécessite une perfusion, et la perfusion nécessite une circulation. Néanmoins, la détection d'une circulation ne veut pas dire qu'il y ait perfusion, et la détection d'une perfusion ne signifie pas nécessairement qu'il y ait fonction. Ainsi, des définitions claires de circulation, de perfusion et de fonction pourraient améliorer la compréhension des tests complémentaires, promouvoir d'autres recherches afin d'identifier un test idéal et standardiser l'utilisation de tests dans les soins aux patients.

Pour illustrer les différences fondamentales entre ces trois paramètres, nous proposons un modèle graphique conceptuel, soit celui d'une fleur arrosée par un boyau d'arrosage (figure). Dans ce modèle, la fleur représente le cerveau et le tronc cérébral. Pour atteindre la fleur, l'eau doit d'abord passer dans le boyau d'arrosage (circulation) puis être acheminée à la fleur (perfusion). Dans le cas d'une MC/DDN, l'important est de déterminer si le cerveau et le tronc cérébral cessent de fonctionner, ce qui équivaut à démontrer que la fleur est morte, indépendamment que l'eau coule dans le boyau ou atteigne la fleur.

Plusieurs articles récents ont passé en revue de manière approfondie les tests complémentaires utilisés pour déterminer un décès neurologique. ${ }^{1,7,10}$ Même après la publication récente des critères de MC/DDN dans le cadre du World Brain Death Project par Greer et collègues, il nous semble que l'utilisation et l'interprétation des tests complémentaires demeurent à la fois mal comprises et controversées. ${ }^{11}$ Nous présentons ici une brève évaluation critique de certains de ces tests, tout en proposant une nouvelle approche conceptuelle et physiologique de la distinction entre circulation, perfusion et fonction 
cérébrales (tableau 1). Nous proposons également des définitions actualisées de la circulation, de la perfusion et de la fonction basées sur notre modèle conceptuel (tableau 2).

\section{Y a-t-il une circulation sanguine cérébrale? (Y a-t-il de l'eau dans le boyau d'arrosage?)}

La circulation est définie comme le mouvement régulier d'un fluide à travers les vaisseaux sanguins tissulaires. La circulation sanguine cérébrale est nécessaire pour la perfusion, et donc pour la fonction. Dans un contexte de MC/DDN, la démonstration sans équivoque de l'absence de circulation est compatible avec la mort neurologique, ${ }^{3}$ mais la présence de circulation en tant que telle ne suffit pas pour prouver qu'il y ait perfusion et fonction cérébrales. L'angiographie conventionnelle à quatre vaisseaux, l'échographie par Doppler transcrânien, et l'angiographie par tomodensitométrie ou résonance magnétique sont les outils traditionnellement employés pour détecter la circulation cérébrale. Bien qu'ayant une bonne sensibilité pour la circulation, ces tests ont néanmoins diverses limitations. Par exemple, des cas de faux négatifs ont été décrits dans lesquels une circulation cérébrale était documentée dans l'angiographie par tomodensitométrie malgré que l'angiographie conventionnelle ait montré une absence d'opacification dans la vasculature cérébrale. ${ }^{12}$ La présence de circulation sanguine (c.-à-d. d'eau dans le tuyau d'arrosage) est relativement facile à démontrer, mais demeure parfois peu concluante; une imagerie supplémentaire est alors nécessaire pour évaluer la perfusion ou la fonction.

\section{En présence de circulation, y a-t-il perfusion cérébrale ? (L'eau atteint-elle la fleur?)}

Alors que la circulation cérébrale est habituellement étudiée dans les principales artères et veines du cerveau, l'évaluation de la perfusion cérébrale implique l'inspection du lit capillaire. Au cours des dernières années, un intérêt croissant s'est manifesté pour l'imagerie de perfusion dans le contexte d'une MC/DDN, mais ces méthodes doivent encore être validées. ${ }^{9,13}$ L'imagerie de perfusion, comme la tomodensitométrie de perfusion, présente de nombreux avantages potentiels par rapport à d'autres modalités de neuroimagerie : elle est largement disponible et dépend moins de l'opérateur; elle pourrait même fournir un diagnostic plus rapide que l'angiographie par tomodensitométrie. $^{13}$ En effet, certains auteurs ont rapporté que la déclaration de $\mathrm{MC} / \mathrm{DDN}$ avait été retardée de plus de 24 heures après une angiographie par tomodensitométrie non concluante, alors qu'ils disposaient de données probantes simultanées de «mort» du tronc cérébral sur la tomodensitométrie de perfusion. ${ }^{13}$ Cela est particulièrement vrai en présence d'une circulation résiduelle de signification incertaine. Alors que l'imagerie de la circulation, telle que l'angiographie par tomodensitométrie, est utile pour évaluer la présence ou l'absence de contraste (circulation) dans les grands vaisseaux cérébraux, l'imagerie de perfusion, telle que la tomodensitométrie de perfusion, a le potentiel de fournir une évaluation quantitative et qualitative du volume et de la circulation sanguins cérébraux dans diverses régions du cerveau. ${ }^{14}$ En outre, la tomodensitométrie de perfusion peut aider à diagnostiquer une catastrophe cérébrale soustentorielle primaire (telle qu'un infarctus du tronc cérébral), une complication relativement rare dans laquelle un déficit de perfusion isolé du tronc cérébral provoque l'arrêt clinique de la fonction du tronc cérébral en dépit de la préservation de la circulation et de la perfusion dans d'autres régions du cerveau. ${ }^{13}$ Sa principale faiblesse à ce jour demeure son manque de validation, mais les études pilotes montrent un excellent profil de sensibilité, de spécificité et de valeurs prédictives par rapport à l'angiographie par tomodensitométrie et à l'examen clinique. ${ }^{13,15,16} \mathrm{La}$ validation à grande échelle de l'exactitude diagnostique de la tomodensitométrie de perfusion pour la MC/DDN est en cours dans le cadre de l'étude prospective INDex-CTP (clinicaltrials.gov : NCT03098511).

\section{S'il y a perfusion, y a-t-il fonction cérébrale? (La fleur} est-elle vivante?)

En tant que cliniciens évaluant les MC/DDN, notre attention se porte principalement sur l'évaluation de la fonction, qui peut être définie comme l'intégration d'une afférence et efférence neuronales continues dans le cerveau et le tronc cérébral en réponse aux stimuli physiologiques. Cette approche est largement acceptée au niveau international et se fonde sur une évaluation clinique documentant l'absence de capacité de conscience et l'aréflexie du tronc cérébral. ${ }^{17}$ En outre, selon les critères récemment publiés du World Brain Death Project 2020 pour la MC/DDN, le diagnostic de décès neurologique ne nécessite que la démonstration de l'absence de fonction du tronc cérébral, essentielle à la conscience et à d'autres fonctions vitales telles que la respiration autonome (tableau 1). ${ }^{11}$ Par conséquent, l'examen physique du tronc cérébral demeure la méthode de référence pour poser un diagnostic de MC/DDN. Malheureusement, cet examen est souvent limité par des facteurs de confusion, tels que l'intoxication et les traumatismes faciaux majeurs, en plus d'être sujet à une 
TABLEAU 1 Comparaison qualitative empirique des tests complémentaires pour la mort cérébrale/le décès déterminé par des critères neurologiques

\begin{tabular}{|c|c|c|c|c|c|}
\hline & \multicolumn{2}{|c|}{ Circulation } & \multicolumn{2}{|c|}{ Perfusion } & \multirow[t]{2}{*}{ Fonction } \\
\hline & Cerveau & Tronc cérébral & Cerveau & Tronc cérébral & \\
\hline Angiographie à 4 vaisseaux & $\oplus$ & $\oplus$ & $\ominus$ & $\ominus$ & $\otimes$ \\
\hline Doppler transcrânien & $\bigoplus$ & $\bigcirc^{*}$ & $\otimes$ & $\otimes$ & $\otimes$ \\
\hline Angiographie par tomodensitométrie & $\oplus \oplus$ & $\bigoplus$ & $\ominus$ & $\otimes$ & $\otimes$ \\
\hline Tomodensitométrie de perfusion & $(\oplus 00)$ & $(\oplus \infty \oplus)$ & $(\oplus \infty \infty)$ & $(\oplus \infty \oplus)$ & $\otimes$ \\
\hline Imagerie par radionucléides & $\bigoplus \oplus$ & $\ominus$ & $\infty$ & $\ominus$ & $\ominus$ \\
\hline Électroencéphalographie & $\otimes$ & $\otimes$ & $\otimes$ & $\otimes$ & $\ominus$ \\
\hline Potentiels évoqués & $\otimes$ & $\otimes$ & $\otimes$ & $\otimes$ & $\ominus$ \\
\hline Examen clinique & $\otimes$ & $\otimes$ & $\otimes$ & $\otimes$ & $\oplus \infty \oplus$ \\
\hline
\end{tabular}

\footnotetext{
$\bigotimes=$ pas utile

$\ominus=$ pourrait être utile (des conditions s'appliquent généralement)

$\bigoplus=$ utile

( ) = à l'étude

* Le Doppler transcrânien peut évaluer la circulation dans les artères vertébrales et l'artère basilaire (si la fenêtre acoustique le permet).

Comparaison qualitative empirique des tests complémentaires et de l'examen clinique pour la mort cérébrale/le décès déterminé par des critères neurologiques. Le nombre de points représente une qualité empirique croissante. La tomodensitométrie de perfusion est actuellement à l'étude dans l'étude prospective INDex-CTP (ClinicalTrials.gov : NCT03098511).
}

variabilité inter-évaluateur. ${ }^{10,18,19} \mathrm{Au}$ fil des ans, de nombreux tests complémentaires se sont concentrés sur l'évaluation fonctionnelle, comme par exemple l'électroencéphalographie, les potentiels évoqués et l'imagerie par radionucléides. La plupart du temps, ces tests fournissent une évaluation objective et qualitative de la fonction corticale cérébrale. Cela dit, leur précision est généralement limitée par les mêmes facteurs de confusion que l'examen clinique. À la lumière de ces limitations, certains auteurs estiment que le test complémentaire idéal consiste à étudier la perfusion cérébrale avec le tomodensitomètre à émission unique de photons $99 \mathrm{mTc}$ HMPAO (SPECT). ${ }^{7}$ Néanmoins, la disponibilité et la résolution spatiale du HMPAO-SPECT sont limitées; de plus, il exige un long temps d'acquisition et est soumis à divers artéfacts qui pourraient également entraver son interprétation, notamment au niveau du tronc cérébral. Un exemple clinique de ces limitations serait un patient présentant un trauma facial (facteur de confusion clinique justifiant des tests complémentaires) hospitalisé dans une unité générale régionale de soins intensifs (donc ayant un accès limité à la neuroimagerie avancée) souffrant d'une hypertension intracrânienne aiguë et une cardiomyopathie neurogénique instable (soit une tolérance limitée à un long temps d'acquisition des images) à la suite d'une hémorragie intracrânienne due à une rupture d'anévrisme basilaire. Un tel patient représenterait toujours un défi considérable; cet exemple illustre la complexité d'une détermination exacte et opportune d'un diagnostic de MC/DDN.

\section{Implications pour l'avenir des tests complémentaires pour les diagnostics de MC/DDN}

Au-delà de l'amélioration de la nomenclature, les distinctions entre circulation, perfusion et fonction ont 
TABLEAU 2 Suggestions de définitions liées au décès déterminé par des critères neurologiques

\begin{tabular}{lll}
\hline & Définition actualisée proposée & Corrélation conceptuelle du modèle \\
\hline Circulation & $\begin{array}{c}\text { Mouvement du sang détecté par opacification des principaux } \\
\text { vaisseaux cérébraux (artères et veines) dans les études sur la } \\
\text { circulation sanguine }\end{array}$ & Y a-t-il de l'eau dans le tuyau d'arrosage? \\
Perfusion & $\begin{array}{l}\text { Circulation au niveau des capillaires dans le tissu cible (dans le cas } \\
\text { présent l'encéphale*, en particulier le tronc cérébral) }\end{array}$ & L'eau atteint-elle la plante? \\
Fonction & $\begin{array}{l}\text { Fonction neuronale clinique organisée du tronc cérébral telle que } \\
\text { démontrée par la conscience et les réflexes du tronc cérébral }\end{array}$ & La fleur est-elle vivante? \\
\hline
\end{tabular}

*L'encéphale est défini comme la combinaison du cerveau et du tronc cérébral.

Proposition de définitions actualisées des concepts de circulation sanguine, de perfusion et de fonction cérébrales à la lumière de notre nouveau modèle conceptuel. Ces définitions actualisées mettent l'accent sur les différences physiologiques entre les trois concepts afin d'éviter une utilisation ou une interprétation erronées de la nomenclature.

des implications pour l'avenir des tests complémentaires dans la détermination de la MC/DDN. Une fois qu'ils auront été minutieusement validés dans des études de précision diagnostique de haute qualité, les tests complémentaires innovateurs qui évaluent la perfusion ( $\mathrm{p}$. ex., tomodensitométrie de perfusion) ou la fonction du tronc cérébral pourraient favoriser une détermination plus rapide de la MC/DDN chez certains patients. Par exemple, ces tests pourraient s'avérer particulièrement utiles pour les patients présentant des lésions cérébrales isolées qui subissent des tests complémentaires pour des indications cliniques (par exemple, des facteurs de confusion à l'examen clinique) ou en raison de règles juridiques (par exemple, l'Allemagne exige des tests complémentaires pour tous les patients soupçonnés de MC/DDN en raison d'une lésion sous-tentorielle). ${ }^{20}$ Comme indiqué précédemment, il existe des données probantes selon lesquelles la circulation sanguine supra-tentorielle pourrait persister chez les patients qui remplissent tous les critères cliniques de MC/DDN et pour lesquels aucune perfusion du tronc cérébral n'est visible par tomodensitométrie de perfusion. ${ }^{13}$ En se fondant sur des rapports isolés et des séries de cas, d'autres auteurs ont émis l'hypothèse que les patients présentant une mort isolée du tronc cérébral évoluaient par la suite à une mort cérébrale complète en raison de l'hypertension intracrânienne due à une hydrocéphalie non communicante (obstructive), aggravée par l'effet de masse sur la veine de Galien. ${ }^{21,22}$ Bien que ce processus puisse se produire en quelques heures chez certains patients, la progression vers une mort cérébrale complète pourrait prendre des semaines chez les patients ayant subi une craniectomie décompressive. ${ }^{22}$ Par conséquent, les tests complémentaires soigneusement validés pour la perfusion ou la fonction du tronc cérébral pourraient permettre un diagnostic rapide de MC/DDN chez de tels patients, évitant ainsi les retards encourus par la nécessité de prouver l'absence de circulation sanguine cérébrale et permettant une évaluation rapide visant un don d'organe.

\section{Conclusion}

Le décès déterminé par des critères neurologiques est fondamentalement une détermination clinique fondée sur l'absence permanente de capacité de conscience et l'aréflexie du tronc cérébral. En présence de facteurs de confusion insolubles ou de l'incapacité de terminer l'évaluation clinique, le recours à des tests complémentaires est essentiel, mais l'interprétation des résultats demeure parfois complexe. Nous proposons un cadre conceptuel permettant de distinguer clairement la circulation, la perfusion et la fonction du cerveau. La fonction cérébrale nécessite une perfusion, et la perfusion nécessite une circulation. Cela dit, la présence d'une circulation ne veut pas dire qu'il y ait perfusion, et la présence d'une perfusion ne signifie pas nécessairement qu'il y ait fonction. Le test complémentaire idéal devrait être rapide, sécuritaire, facilement disponible, non invasif, peu coûteux et non susceptible aux facteurs de confusion. ${ }^{7}$ En outre, le test idéal devrait être aussi proche que possible d'une évaluation de la fonction sans facteur de confusion et devrait s'abstenir d'utiliser la circulation comme substitut. Un tel test idéal n'a pas encore été validé.

\section{Disclosures None.}

Funding statement None.

Editorial responsibility This submission was handled by Dr. Alana M. Flexman, Associate Editor, Canadian Journal of Anesthesia.

Déclaration Aucune.

Déclaration de financement Aucune. 
Responsabilité éditoriale Cet article a été traité par Dre Alana M Flexman, rédactrice adjointe, Journal canadien d'anesthésie.

Open Access This article is licensed under a Creative Commons Attribution-NonCommercial 4.0 International License, which permits any non-commercial use, sharing, adaptation, distribution and reproduction in any medium or format, as long as you give appropriate credit to the original author(s) and the source, provide a link to the Creative Commons licence, and indicate if changes were made. The images or other third party material in this article are included in the article's Creative Commons licence, unless indicated otherwise in a credit line to the material. If material is not included in the article's Creative Commons licence and your intended use is not permitted by statutory regulation or exceeds the permitted use, you will need to obtain permission directly from the copyright holder. To view a copy of this licence, visit http://creativecommons.org/licenses/ by-nc/4.0/.

\section{References}

1. Kramer AH. Ancillary testing in brain death. Semin Neurol 2015; 35: 125-38.

2. Shemie SD, Doig C, Dickens B, et al. Severe brain injury to neurological determination of death: Canadian forum recommendations. CMAJ 2006; 174: S1-12.

3. Shemie SD, Lee D, Sharpe M, Tampieri D, Young B; Canadian Critical Care Society. Brain blood flow in the neurological determination of death: Canadian expert report. Can J Neurol Sci 2008; 35: 140-5.

4. Shemie SD, Hornby L, Baker A, et al. International guideline development for the determination of death. Intensive Care Med 2014; 40: 788-97.

5. Bernat JL, Capron AM, Bleck TP, et al. The circulatoryrespiratory determination of death in organ donation. Crit Care Med 2010; 38: 963-70.

6. Anonymous. A definition of irreversible coma. Report of the Ad Hoc Committee of the Harvard Medical School to examine the definition of brain death. JAMA 1968; 205: 337-40.

7. Heran MK, Heran NS, Shemie SD. A review of ancillary tests in evaluating brain death. Can J Neurol Sci 2008; 35: 409-19.

8. Wijdicks EF, Varelas PN, Gronseth GS, Greer DM; American Academy of Neurology. Evidence-based guideline update: determining brain death in adults: report of the Quality Standards Subcommittee of the American Academy of Neurology. Neurology 2010; 74: 1911-8.
9. Shankar JJ, Banfield JC. Comments on Shemie et al.: international guideline development for the determination of death. Intensive Care Med 2015. https://doi.org/10.1007/s00134015-3679-3.

10. Greer DM, Wang HH, Robinson JD, Varelas $P N$, Henderson $G V$, Wijdicks EF. Variability of brain death policies in the United States. JAMA Neurol 2016; 73: 213-8.

11. Greer DM, Shemie SD, Lewis A, et al. Determination of brain death/death by neurologic criteria: the world brain death project. JAMA 2020; 324: 1078-97.

12. Combes JC, Chomel A, Ricolfi F, d'Athis P, Freysz M. Reliability of computed tomographic angiography in the diagnosis of brain death. Transplant Proc 2007; 39: 16-20.

13. Shankar JJ, Vandorpe R. CT perfusion for confirmation of brain death. AJNR Am J Neuroradiol 2013; 34: 1175-9.

14. MacDonald D, Stewart-Perrin B, Shankar JJ. The role of neuroimaging in the determination of brain death. J Neuroimaging 2018; 28: 374-9.

15. Shankar JJ, Stewart-Perrin B, Quraishi AU, Bata I, Vandorpe R. Computed tomography perfusion aids in the prognostication of comatose postcardiac arrest patients. Am J Cardiol 2018; 121: 874-8.

16. Shankar JJ, Green R, Virani K, Wong H, Eddy K, Vandorpe R. Admission perfusion CT for classifying early in-hospital mortality of patients with severe traumatic brain injury: a pilot study. AJR Am J Roentgenol 2020; 214: 872-6.

17. Lewis A, Bakkar A, Kreiger-Benson E, et al. Determination of death by neurologic criteria around the world. Neurology 2020. https://doi.org/10.1212/wnl.0000000000010541.

18. Shappell CN, Frank JI, Husari K, Sanchez M, Goldenberg F, Ardelt A. Practice variability in brain death determination: a call to action. Neurology 2013; 81: 2009-14.

19. Pandey A, Sahota P, Nattanmai P, Newey CR. Variability in diagnosing brain death at an academic medical center. Neurosci J 2017. https://doi.org/10.1155/2017/6017958.

20. Hoffmann $O$, Masuhr $F$. Use of observational periods or ancillary tests in the determination of brain death in Germany. Eur Neurol 2015; 74: 11-7.

21. Marcellino $C$, Braksick SA, Wijdicks EF. How does the brain die after a massive posterior fossa lesion? Neurocrit Care 2020. https://doi.org/10.1007/s12028-020-01147-3.

22. Varelas $P N$, Brady $P$, Rehman $M$, et al. Primary posterior fossa lesions and preserved supratentorial cerebral blood flow: implications for brain death determination. Neurocrit Care 2017; 27: 407-14.

Publisher's Note Springer Nature remains neutral with regard to jurisdictional claims in published maps and institutional affiliations. 